\title{
Hybrid Recommendation Algorithm for Potential Features of Users in Social Networks Based on Swarm Intelligence
}

\author{
Lu Xing-Hua ${ }^{1, a *}$, Liu Ming-Yuan ${ }^{1}$,Huang Hao-Hong ${ }^{1}$ and Wu Hong-Yu ${ }^{1}$ \\ ${ }^{1}$ Huali College Guangdong University of Technology, 511325 Guangdong Guangzhou, China
}

\begin{abstract}
The intelligent recommendation ability of social networks is improved, a hybrid recommendation algorithm is proposed based on swarm intelligence for users' potential features in social networks. The feature extraction model of social network users is constructed, and the potential features and associated information of social network users are divided by swarm intelligence optimization technology, and the user features are learned by swarm intelligence and association rules mining. The related information of recommended items in social network is obtained, and the improvement of user item feature recommendation algorithm of social network is realized. The simulation results show that the proposed algorithm can effectively improve the accurate delivery rate of user feature recommendation in social network, and the hybrid recommendation ability for user behavior is strong, the network overhead is stable and the performance is superior
\end{abstract}

\section{Submitting the manuscript}

With the popularity of the network in society, the combination of network and people's real life becomes more and more closely, people can easily get information in the network, education can also be expanded from the network mode. Based on this development situation, people's ideas are very vulnerable to social networks, especially for young students, social networks. In order to make the social network better applied, the relevant departments should restrict the individual behavior of the Internet users from the psychological perspective. In the network age, people's daily life and work mode have changed greatly, the channels for people to obtain information are no longer fixed, with the Internet, People can easily understand the information they want to understand, not only to meet the needs of people, but also to provide a certain degree of satisfaction in quality, the rich and diversified sources of information, to promote the change of people's cognitive style, because the social network public opinion is easy to spread, the user characteristics of social networks have attracted great attention $^{[1]}$. The mainstream media will guide it to attract people's attention, and the credibility of the mainstream media can improve the reliability of the public opinion in micro-blog. The social network has the characteristics of openness and self-organization. The user characteristics recommendation in social network is the key to ensure the security of users and the protection of privacy. It has great significance for designing of recommendation models and algorithms for user characteristics in social networks ${ }^{[2]}$.

At present, there are fuzzy ant colony search algorithm, particle swarm optimization recommendation algorithm and social network spatial area search recommendation algorithm for user potential feature recommendation algorithm in social network. Particle swarm optimization (PSO) algorithm is used to recommend the user features of social networks, and some research results have been achieved $^{[3]}$. In reference [4], a user feature recommendation algorithm based on extended recommendation of semantic related data is proposed. Fuzzy decision making and mathematical modeling are used to first recommend the construction of model, and particle swarm optimization is used to realize information location. Improve the accuracy of recommendation algorithm, but the algorithm may lead to the disclosure of user source information, the security is not good. In reference [5], a new feature recommendation algorithm for social network users based on multi-agent negotiation trust analysis is proposed, in which the fixed features in social network are segmented by swarm intelligence, and the recommendation accuracy is improved. However, the algorithm has the problem of large amount of computation. Comprehensive analysis shows that the traditional social network user feature recommendation algorithm uses agent negotiated cloud trust data recommendation, which is subjective and poor in performance to the abnormal user feature recommendation of social network.

The disadvantages of traditional methods need to be solved, a user potential feature recommendation algorithm based on swarm intelligence is proposed to improve the ability of user feature recommendation and detection in social network. Firstly, the user feature extraction model of social network is constructed, and the signal model is simulated and constructed. The fuzzy decision method is used to detect and judge the features of users, and the accurate detection and recommendation of user features are realized. Finally, simulation experiments are carried out to demonstrate the effectiveness and superiority of the proposed algorithm.

*Corresponding author: ${ }^{a}$ xhlu@gdut.edu.cn 


\section{Social network distributed architecture and user feature extraction}

\subsection{Social network distributed architecture}

According to the four-stage model of interest development proposed by Hidi and Renninger ${ }^{[6]}$, the users of interest social network service platform are divided into situational interest users, individual interest users and group interest users according to the level of interest. Situational interest users are those who, for some reasons, have a desire to understand a certain interest and engage in exploratory activities. Such users are the most numerous and unstable in the user population. How to turn it into an individual interest user will be one of the main issues to be considered in design ${ }^{[7]}$. The individual interest user refers to those interested social users who gradually stabilize and develop because of the surrounding environment support, individual interest is concerned, and their own efforts are made. Such users are the backbone of the user community, and it is important to maintain their level of activity and innovation on the platform. Group interest user refers to the interest circle users who promote each other because of the interest connection between individual interest users. Common social platforms such as QQ, WeChat, Weibo and MSNs are defined as social networks where users with similar interests or commonalities are connected via Internet networks or mobile phones. The social network structure of mobile terminal interconnection is obgiend ${ }^{[8]}$, and the architecture of distributed social network is shown in figure 1 .

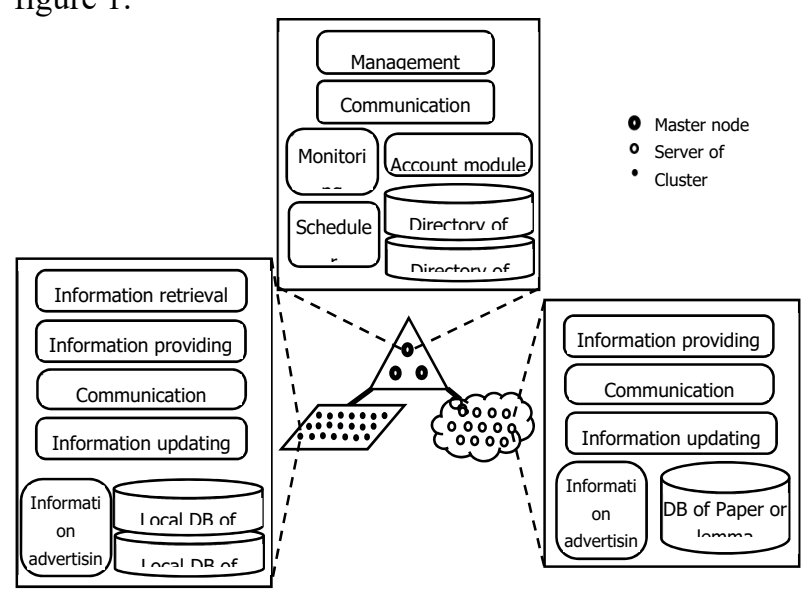

Fig.1 Mobile social network distributed structure

The distributed mobile social network architecture shown in figure 1 is based on the Web mobile social network (Web-based MSNs, WMSNs) and the decentralized mobile social network (Decentralized MSNs, DMSNs) ${ }^{[9]}$, using cloud computing systems to provide cloud-based services. For developers to use when creating applications. With the development of network technology, mobile social network has become the general trend of information processing. Based on the new SAS application that can be created by cloud computing system, the MSNs mobile social network platform is realized, and the program control extension and intelligent sharing method of cloud computing system are used to realize the information exchange and storage ${ }^{[10]}$.

In the design of mobile social network architecture, the routing node distribution model of social network needs to be constructed, and the connections of nodes in the network are discontinuous. Suppose there were $n$ recommended negotiations $(n>1)$ between nodes A and B of social network, where the number of success was $a$ ( $a>1$, and the communication time was $t$, the message set of social network node $A$ is defined as $S_{m s g}^{(A)}(t)$, the user characteristic set of social network is $s_{c t x t}^{(A)}(t)$. The information recommends trust inertia weight is Ackley function, as follows:

$f_{6}(x)=\operatorname{SINR}-20 \exp \left(-0.2 \sqrt{\frac{1}{D} \sum_{d=1}^{D} x_{d}^{2}}\right)-\exp \left(\frac{1}{D} \sum_{d=1}^{D} \cos \left(2 \pi x_{d}\right)\right)$

Where, $\operatorname{SINR}$ is the threshold value, $\beta \geq 1$, the path loss coefficient is $\alpha \in(2,6)$, and the chance routing follows the rules:

$$
\begin{aligned}
& S_{m s g}^{(i)}(t+\Delta t)=f\left(S_{m s g}^{(A)}(t), S_{m s g}^{(1)}(t), \ldots, S_{m \mathrm{sg}}^{(n)}(t), S_{c t x t}^{(1)}(t), \ldots, S_{c x t}^{(n)}(t)\right), \forall i \in\{A, 1, \ldots, n\} \\
& S_{c t x t}^{(i)}(t+\Delta t)=f\left(S_{c t x t}^{(A)}(t), S_{c t x t}^{(1)}(t), \ldots, S_{c t x t}^{(n)}(t)\right), \forall i \in\{A, 1, \ldots, n\}
\end{aligned}
$$

The potential characteristics and associated information of social network users are divided into several edges as $\ell=r / \sqrt{2}$ by the method of swarm intelligence optimization technology, and the routing topology of social networks is $G(V, r), \quad\left(r \leq \delta \cdot r_{\max }\right.$, $\left.\delta \in(0,1), r_{\max }=\left(P\left(N_{0} \beta\right)^{-1}\right)^{1 / \alpha}\right)$, where $e_{i j}$ represents the edges connected to social groups $G_{i}$ and $G_{j}$ in the network. A routing protocol model is constructed to provide a network model for the recommendation of user characteristics in social networks ${ }^{[11]}$.

\subsection{Feature modeling of recommendation information}

On the basis of the above network model design, in order to realize the effective recommendation of user characteristics, signal modeling is the key. The signal detection method is used to implement information recommendation. The signal model characteristic modeling process of the recommended information is described as follows ${ }^{[12]}$. Given the social network $G=(V, E)$, the node $v$ is any node in the network, that is, $v \in V$, each node produces each node. A forwarding number $f w d(m) \geq 1$ is generated at the same time, the node betweenness of the social network is calculated to obtain the directional spatial search model of the recommended signal:

$$
H(z)=\frac{1+a z^{-1}+z^{-2}}{1+a r z^{-1}+r^{2} z^{-2}}, 0<r<1
$$

Where, $r$ represents the internal node, $H(z)$ represents the spatial clustering center of the user's characteristics, and the linear expression of the envelope extension state equation of the signal detection system is obtained as follows: 


$$
H(z)=\frac{1+\sin \theta_{2}}{2} \frac{1+2 \sin \theta_{1} z^{-1}+z^{-2}}{1+\sin \theta_{1}\left(1+\sin \theta_{2}\right) z^{-1}+\sin \theta_{2} z^{-2}}
$$

In the above formula, $H(z)$ denotes the function of user feature recommendation system, $\theta_{1}$ is the angle of recommendation center vector offset of node $n$, and $\theta_{2}$ indicates the angle of recommendation center vector offset of node $m$. In order to realize the main feature modeling and feature extraction of data, the feature extraction of user features is carried out, and then the information flow vector model is described as:

$$
x=\sum_{i=1}^{N} s_{i} \Psi_{i}=\Psi S, \Psi=\left[\Psi_{1}, \Psi_{2}, \ldots, \Psi_{N}\right]
$$

Where, $s_{i}$ is a resource information request signal. In order to obtain a smaller response time and realize user feature recommendation, the number of shortest paths passing through each side of a social network, $B(e)$, $B(e)=\sum_{v_{i} \neq v_{j} \in V} n_{i j}(e), n_{i j}(e)$ is used to represent the optimal coverage between $v_{i}, v_{j} \in V$ nodes. The separation function of self-organizing social network is expanded by the separation series near $\hat{X}(k \mid k)$. The results are expressed as follows:

$$
\begin{aligned}
& X(k+1)=f(\hat{X}(k \mid k))+ \\
& f_{X}(k)[X(k)-\hat{X}(k \mid k)]+O+V(k)
\end{aligned}
$$

From this, the signal model feature modeling of recommendation information is obtained, which provides accurate original information basis for information recommendation $^{[13]}$.

\section{Algorithm implementation and key technology description}

Based on the above network model construction and signal feature extraction, the user feature detection and recommendation algorithm of social network is designed. Because the traditional user feature recommendation algorithm of social network adopts the cloud information negotiated by the main body. Any data recommendation, the social network features of abnormal user recommendation is subjective, poor performance ${ }^{[14]}$. In order to overcome the disadvantages of traditional methods, this paper proposes a user potential feature recommendation algorithm based on swarm intelligence in social networks. Combined with the characteristics of spectrum and energy changing with time, the user characteristics are studied by swarm intelligence learning and association rules mining. The association information of recommended items in social network is expressed as follows:

$$
P_{S}=p_{2 \mathrm{D}}{ }^{k}\left(1-p_{2 \mathrm{D}}\right)^{N-1-k} \sum_{i=1}^{\infty} \lambda_{S}^{i}=\frac{\lambda_{S}}{1-\lambda_{S}}
$$

Where, $\lambda_{s}$ is the training sequence OFDM training length, $p_{2 \mathrm{D}}$ is the adaptive frequency flat-top offset probability of the recommendation information of social network under abnormal interference. By fuzzy decision and dynamic fusion design, the mixing of user features is obtained. The reverberation data is a set of locally stationary Rayleigh color noise. The ambiguity function of aliasing spectrum under different scale factors corresponds to the output of different time-delay related processors in this scale ${ }^{[15]}$. Fuzzy decision can be adopted. In a deterministic decision method, the starting time $T_{0}$ is calculated to receive the pseudo code sequence through the link layer, and the channel bandwidth of the user characteristic communication in the social network is obtained as follows:

$$
\text { ITrust }_{a \rightarrow c}=\frac{\sum_{b \in a d j(a, c)} \text { DTrust }_{a \rightarrow b} \times\left(\text { DTrust }_{b \rightarrow c} \times \beta_{d}\right)}{\sum_{b \in a d j(a, c)} \text { DTtrust }_{a \rightarrow b}}
$$

In the user feature recommendation, the spectrum $Z$ is detected from the Rayleigh distribution of the parameter $\beta_{d}$, where:

$$
\beta_{d}=(M P D i s t-d+1) / M P D i s t, d \in[2, M P D i s t]
$$

Where, $\operatorname{adj}(a, c)$ denotes the number of $a \rightarrow c$ paths. When $\beta_{d} \in(0,1]$, the following triangular formula is applied to the adaptive multi-source fading path of social network transmission channel:

$$
\frac{1}{\tan \alpha}=\frac{\cos \alpha}{\sin \alpha}=\frac{1-2 \sin \left(\frac{\alpha}{2}\right) \cos \left(\frac{\alpha}{2}\right) \tan \left(\frac{\alpha}{2}\right)}{2 \sin \left(\frac{\alpha}{2}\right) \cos \left(\frac{\alpha}{2}\right)}=\frac{1}{\sin \alpha}-\tan \left(\frac{\alpha}{2}\right)
$$

In the upper formula, $\alpha$ is the aliasing spectrum ambiguity function of the master-slave transmission channel, and $g_{\alpha}{ }^{\prime}(u)$ represents the pulse of the social network transmission channel. The fuzzy decision method is used to detect and judge the characteristics of the user, so as to realize the accuracy of the user characteristics. Detection and recommendation, in the process of fuzzy decision, there are two models of social network user feature recommendation and inter-community user feature recommendation. The distribution uses fuzzy feature segmentation method to segment user features ${ }^{[16]}$.

\section{SIMULATION EXPERIMENT AND RESULT ANALYSIS}

In order to test the performance of this algorithm in the realization of social network user feature recommendation, a simulation experiment is carried out. The experiment environment is: Myeclipse 8.0 experimental simulation platform and java platform development language, combined with swarm package, the computer is Core i33220CPU, 4G memory, Win10 operating system. The parameters in the design of mobile social network user feature recommendation system are as follows: $\rho_{S M}=50$, $A_{S M}=6, D_{S M}=5, C_{m}=1, g_{N a}=3, g_{K}=5$, $g_{L}=0.05, g_{T}=5, E_{N a}=50, E_{K}=-90, E_{L}=-70$, $E_{T}=120$. The maximum communication distance between nodes of social network is $1000 \mathrm{~m}$. The minimum 
communication distance is $R_{\min }=10 \mathrm{~m}$. Social network nodes communicate in 100th round, bandwidth is $1 \mathrm{Mb}$, packet size is $256 \mathrm{~b}$. the network has 140 communication nodes and 10 bug nodes, the speed of data communication is $255 \mathrm{kBps} / 10 \mathrm{MBps}$, the single message stranded node cache points. Based on the above simulation environment and parameter design results, the user characteristics are recommended. Firstly, the signal model is constructed, and the simulation results of the user characteristic signal model in the social network are obtained as shown in figure 2 .

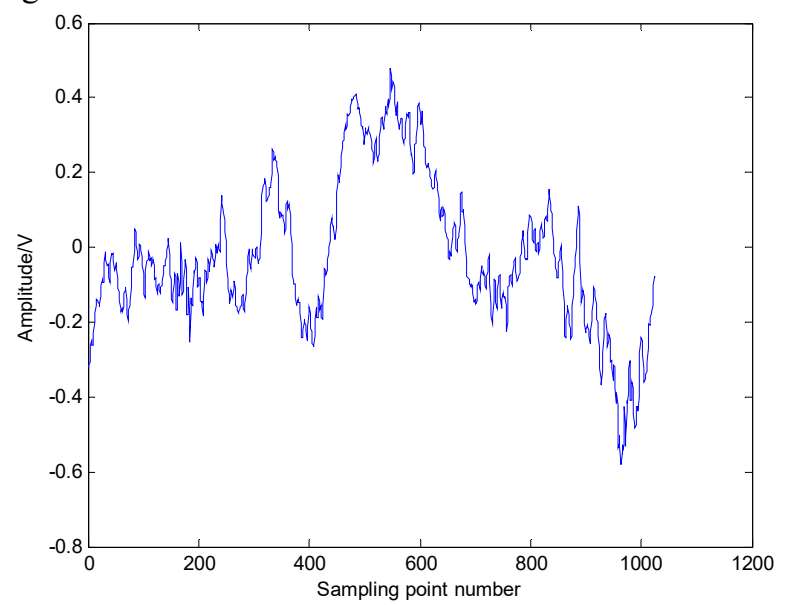

(a) sample 1

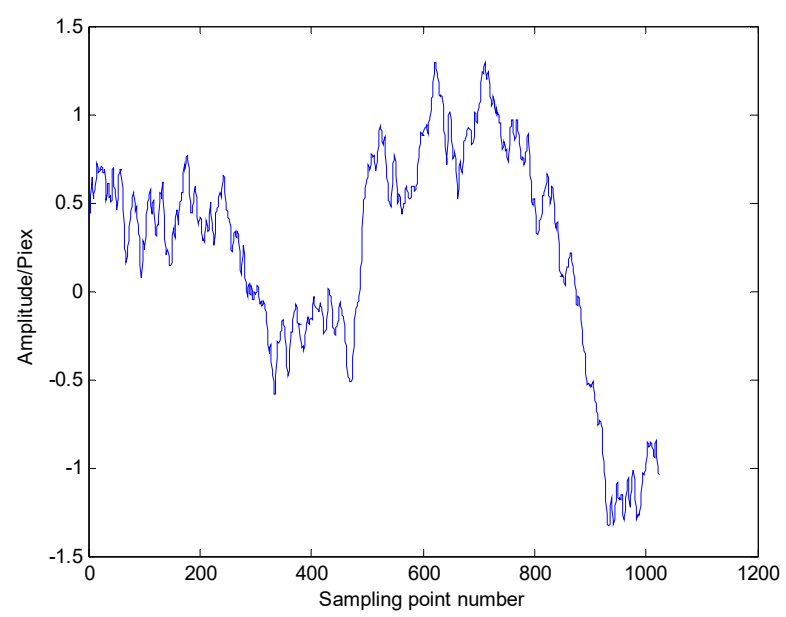

(b) sample 2

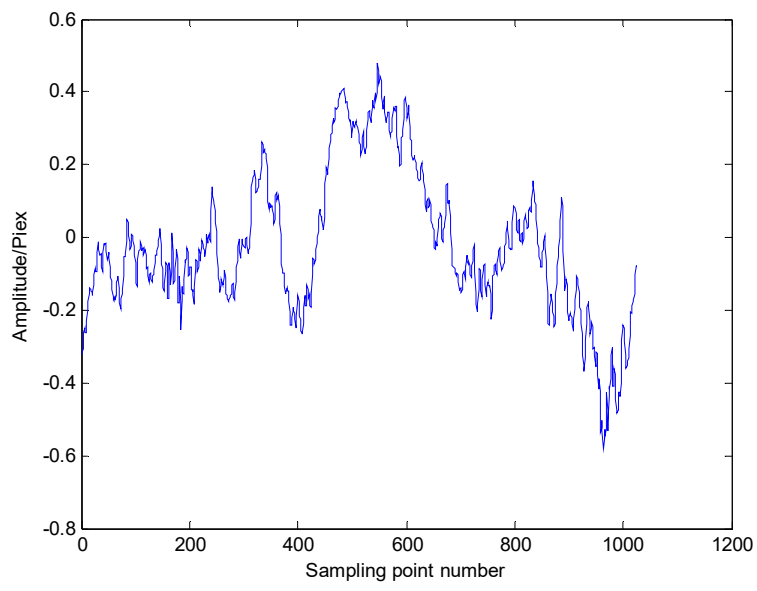

(c) sample 3

Fig.2 Social network user feature information collection output samples

It can be seen from the diagram that the user features in the original social network are annihilated in the external interference noise, and it is difficult to realize effective signal detection and user feature recommendation. It is necessary to carry out signal detection and recommendation algorithm design. The signal detection and user feature recommendation algorithm are designed and simulated, in order to design and simulate the user feature recommendation algorithm. Comparing the performance of the algorithm, this algorithm and the traditional method, with the accurate delivery rate recommended by the information as the test index, get the accurate recommendation rate comparison structure under the different social network cache scale, as shown in Figure 3.

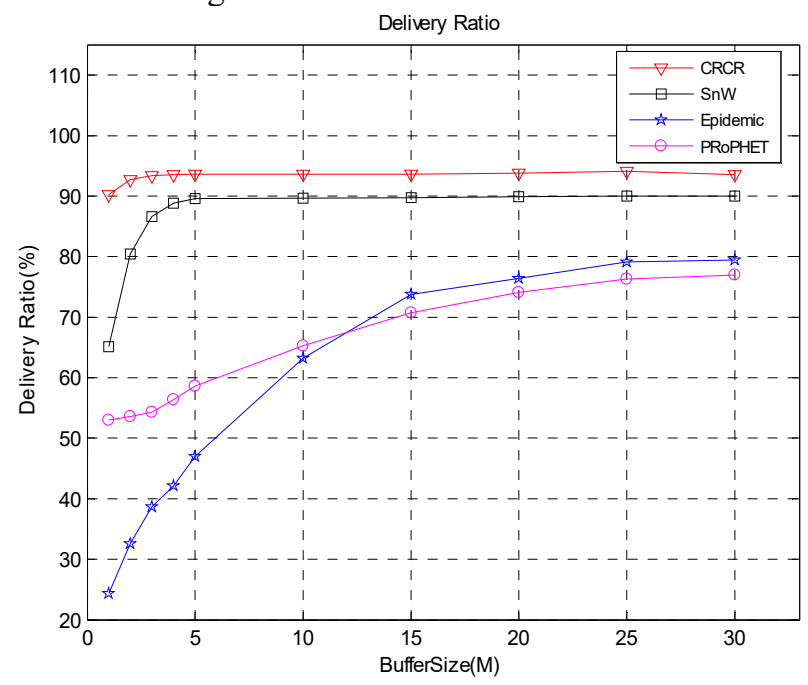

Fig. 3 User potential feature recommendation accurate delivery rate in social networks

It can be seen from the figure that the proposed algorithm has a higher user feature recommendation accurate delivery rate. With the increase of cache space, the number of messages delivered successfully increases, and the network overhead of this algorithm is stable, which is only 6.4, compared with the traditional algorithm. The method is higher than 10, which shows that the hybrid 
recommendation ability of the proposed algorithm in realizing user feature recommendation is strong, and the accuracy of recommendation is improved.

\section{CONCLUSIONS}

In this paper, a hybrid recommendation algorithm based on swarm intelligence for users' potential features in social networks is proposed. The feature extraction model of social network users is constructed, and the potential features and associated information of social network users are divided by swarm intelligence optimization technology, and the user features are learned by swarm intelligence and association rules mining. The related information of recommended items in social network is obtained, and the improvement of user item feature recommendation algorithm of social network is realized. The simulation results show that the proposed algorithm can effectively improve the accurate delivery rate of user feature recommendation in social network, and the hybrid recommendation ability for user behavior is strong, the network overhead is stable and the performance is superior. This method has good application value in the independent recommendation design of social network.

\section{ACKNOWLEDGMENTS}

This project is supported by and 2018 Undergraduate Scientific and Technological Innovation Project Fund of Guangdong Province (pdjhb0634).

\section{REFERENCES}

1. E. Bulut, B.K. Szymanski. Exploiting Friendship Relations for Efficient Routing in Mobile Social Networks[J]. IEEE Transactions on parallel and distributed systems.2012,23(12):2254-2265

2. A. Khosravi and J. Pan. Exploring personal interest in intermittently connected wireless mobile social networks [A]. In IEEE Consumer Communications and Networking Conference (CCNC2011) [C], 2011, pp503-508

3. N. Kayastha, W. Ping, et al. Applications, Architectures, and Protocol Design issues for Mobile Social Networks: A Survey[J]. Proceedings of the IEEE. 2011,99(12): 2130-2158

4. YANG Fengrui, ZHENG Yunjun, ZHANG Chang. Hybrid recommendation algorithm based on probability matrix factorization. Journal of Computer Applications, 2018, 38(3): 644-649.

5. SHEN Xueli, QIN Shujuan. Anomaly detection based on synthetic minority oversampling technique and deep belief network. Journal of Computer Applications, 2018, 38(7): 1941-1945.

6. YANG Y H, HUANG H Z, SHEN Q N, et al. Research on intrusion detection based on incremental GHSOM[J]. Chinese Journal of Computers, 2014, 37(5):1216-1224.
7. CHEN H, WAN G X, XIAO Z J. Intrusion detection method of deep belief network model based on optimization of data processing $[\mathrm{J}]$. Journal of Computer Applications, 2017, 37(6):1636-1643.

8. YIN C L, ZHU Y F, FEI J L, et al. A deep learning approach for intrusion detection using recurrent neural networks[J]. IEEE Access, 2017, 5:2195421961.

9. GAO N, HE Y Y, GAO L. Deep learning method for intrusion detection in massive data[J]. Applications Research of Computers, 2018,35(4):1197-1200.

10. ZHOU Yuhao, ZHANG Hongling, LI Fangfei, QI Peng. Local focus support vector machine algorithm. Journal of Computer Applications, 2018, 38(4): 945948.

11. ZHANG Y, LI Z R, LIU X D. Active learning SMOTE based imbalanced data classification[J]. Computer Applications and Software, 2012, 29(3):91-93.

12. CAO J X, DONG D, XU S, et al. A k-core based algorithm for influence maximization in social networks[J]. Chinese Journal of Computers, 2015, 38(2):238-248.

13. WANG C, CHEN W, WANG Y. Scalable influence maximization for independent cascade model in large-scale social networks[J]. Data Mining \& Knowledge Discovery, 2012, 25(3):545-576

14. NING Xueli, LUO Yonglong, XING Kai, ZHENG Xiaoyao. Frequent location privacy-preserving algorithm based on geosocial network. Journal of Computer Applications, 2018, 38(3): 688-692.

15. YE A Y, LI Y C,MA J F, et al. Location privacypreserving method of $\mathrm{k}$-anonymous based-on service similarity[J]. Journal on Communications, 2014, 35(11):162-169.

16. WU Zheng, YU Hongtao, LIU Shuxin, ZHU Yuhang. User identification across multiple social networks based on information entropy. Journal of Computer Applications, 2017, 37(8): 2374-2380. 